\title{
sciendo
}

10.2478/AMB-2021-0009

\section{A STUDY OF PARATYPHOID FEVER IN BULGARIAN CHILDREN}

\author{
M. Pavlova', E. Alexandrova', Y. Kalchev'², V. Velev, M. Murdjeva²,3, T. Kantardjiev \\ ${ }^{1}$ National Center of Infectious and Parasitic Diseases (NCIPD) - Sofia, Bulgaria \\ ${ }^{2}$ Faculty of Pharmacy, Medical University - Plovdiv, Bulgaria \\ 3University Hospital "Sv. George" - Plovdiv, Bulgaria \\ ${ }^{4}$ University Hospital for Infectious and Parasitic Diseases "Prof. Ivan Kirov", Medical University - Sofia, Bulgaria
}

\begin{abstract}
Objective. To study both the molecular discrimination of D-tartrate fermenting and non-fermenting strains of Salmonella enterica subsp. enterica serotype Paratyphi B isolated from patients with paratyphoid fever and the clinical course of this disease. Materials and methods. The isolates examined were from children aged 3 months to 9 years. A total of 33 Salmonella strains were serotyped as Salmonella Paratyphi B, with an antigenic formula based on O- and $\mathrm{H}$ - antigens: 1,4, [5], 12: b: 1,2 by Kauffmann-White classification. Results. Multiplex PCR analysis confirmed all tested strains as $d$-tartrate fermenting $(d T+)$, also referred to as variant Java. Discussion. We found that the most common cause of paratyphoid fever among children in Bulgaria is variant Java Salmonella Paratyphi B. Most children had classic symptoms of acute gastroenteritis - fever, watery diarrhea and vomiting.
\end{abstract}

Key words: Salmonella Paratyphi B, d-tartrate fermenters, multiplex PCR, paratyphoid fever

Corresponding author: V. Velev, MD, PhD, Medical University, 17 Blvd. "Geshov", Bg - 1000 Sofia, tel.: 0889563412, E-mail:Velev_md@abv.bg

\section{INTRODUCTION}

$\mathrm{P}$ aratyphoid fever caused by Salmonella serotypes Paratyphi A, B and C is an infectious disease prevalent mainly in developing countries, while in developed countries it is most commonly observed in people returning from travel to endemic areas [1, 2]. Paratyphoid fever is a multi-systemic disease characterized by persistent fever, diarrhea or constipation, abdominal pain, chills, immunological complications [3]. Bacteriological confirmation of this disease is based on the isolation of the microorganism from feces or blood $[4,5,6]$. In the last five years, between 15 and 20 cases of paratyphoid fever have been registered in Bulgaria annually [3].

The subject of many studies is S. enterica subsp. enterica serovar Paratyphi B and variant S. Paratyphi B, previously referred to as Salmonella Java. These are biovars of common serotype 1,4, [5], 12: b: 1,2, which makes it impossible to distinguish virulent agents by the use of serotyping methods. Salmonella enterica subspecies enterica serovar Paratyphi B can be differentiated by the fermentation of dextrorotatory $[\mathrm{L}(+)]$-tartrate (d-tartrate), using a laborious process and complex biochemical tests. The main difference between them is that S. Paratyphi B are d-tartrate- 
non-fermenting (dT-) strains and biovar Java are dtartrate-fermenting $(\mathrm{dT}+)$ strains $[7,8,9]$.

\section{OBJECTIVE}

To apply and describe a fast and reliable PCR-based method for discrimination of S. Paratyphi B (dT-) and variant Java Salmonella $(\mathrm{dT}+)$ strains and to analyze the clinical course of paratyphoid fever among Bulgarian children.

\section{MATERIALS AND METHODS}

\section{Bacterial strains and serotyping}

The study included 33 Salmonella strains, representing S. enterica subsp. enterica serovar Paratyphi B isolated in the period from January 2017 to November 2018 from feces of patients with paratyphoid fever between 3 months and 9 years of age. 15 (15/33) strains from the University Hospital for Infectious Diseases, Sofia, and 18 (18/33) strains from the University Hospital "Sveti Georgi", Plovdiv were submitted to the National Reference Laboratory of Enteric Pathogens at the National Center of Infectious and Parasitic Diseases (NCIPD) for microbiological confirmation.

All strains were confirmed by cultivation on Deoxycholate Citrate Agar, biochemical tests (API $20 \mathrm{E}$, Biomerieux), OB agglutination: 1,4, [5], 12; $\mathrm{H} 1$ phase: b and $\mathrm{H} 2$ phase: 1,2-antisera (SSI, Denmark; Sifin, Germany and SSI, Copenhagen, Denmark) according to the White-Kauffmann-Le Minor scheme. The strains were stored at $-80^{\circ} \mathrm{C}$ until further use.

\section{Antimicrobial susceptibility testing}

Antimicrobial susceptibility was investigated by Bauer-Kirby disc diffusion method to cefotaxime $(30 \mu \mathrm{g})$, cefoxitin $(30 \mu \mathrm{g})$, ceftazidime $(30 \mu \mathrm{g})$, ampicillin (10 $\mu \mathrm{g})$, amoxicillin/ clavulanic acid $(20 / 10 \mu \mathrm{g})$, amikacin $(30 \mu \mathrm{g})$, gentamicin $(10 \mu \mathrm{g})$, tetracycline $(30 \mu \mathrm{g})$, chloramphenicol $(30 \mu \mathrm{g})$, ciprofloxacin $(5 \mu \mathrm{g})$, nalidixic acid $(30 \mu \mathrm{g})$, trimethoprim/sulfamethoxazole $(1,25 /$ 23,75 $\mu \mathrm{g}$ ) (EUCAST-2018) [10].

\section{PCR-based method for confirmation of S. Para- typhi B}

Bacterial DNA was extracted using PrepManTM (Applied Biosystems,Foster City, CA) in accordance with manufacturer's instructions. The extracted DNA was stored at $-20^{\circ} \mathrm{C}$ until used in the $\mathrm{mPCR}$.

Multiplex PCR was carried out using 2 sets of primers. Primer ST11 (5'-AGC CAA CCA TTG CTA AAT TGG CGCA- 3') and ST15 (5'-GGT AGA AAT TCC CAG CGG GTA CTG-3') - were genus specific for
Salmonella. The resulting PCR product size for these primers was 429 bp. The second primer pair 167 (5' -CAC ATT ATT CGC TCA ATG GAG- 3') and 166 (5'-GTA AGG GTA ATG GGT TCC- 3') were used to detect the presence or absence of ATG start codon for the gene encoding the putative cation transporter in the S. Paratyphi B, resulting in a 290-bp PCR fragment if the strain was d-tartratepositive [11].

PCR analysis was optimized to run in a final volume of $25 \mu \mathrm{l}$ reaction at these primers and the following conditions - 1X PCR buffer with $\mathrm{MgCl}$; dNTP 0,2 $\mathrm{mM} ; 0.04 \mathrm{U} / \mu \mathrm{l}$ SuperHotTaq DNA pol.; $0.4 \mu \mathrm{M}$ of primers 166 and 167, $0.1 \mu \mathrm{M}$ of primers ST11 and ST151 and $2 \mu \mathrm{l}$ of the DNA template. A negative control containing the same reaction mixture except the DNA template was included in every experiment and E. coli ATCC 25922 as a second one.

An initial denaturation at $95^{\circ} \mathrm{C}$ for 3 min was followed by 27 cycles of denaturation at $95^{\circ} \mathrm{C}$ for $30 \mathrm{sec}$, annealing at $62^{\circ} \mathrm{C}$ for $1 \mathrm{~min}$ and extension at $72^{\circ} \mathrm{C}$ for $1 \mathrm{~min}$. Finally, an additional extension was achieved for $5 \mathrm{~min}$ at $72^{\circ} \mathrm{C}$. A $10 \mu \mathrm{l}$ aliquot of each PCR product was electrophoresed on a $1.5 \%$ agarose gel and visualized and photographed under UV illumination.

\section{RESULTS AND DISCUSSION}

Thirty-three cases of infection with S. Paratyphi B were studied in order to analyse paratyphoid fever in the southern and western regions of Bulgaria (city of Sofia and city of Plovdiv). All thirty-three S. Paratyphi $B$ isolates showed typical growth and biochemical characteristics and were both $O B$ positive and $H: b$ and $\mathrm{H}: 1.2$ positive according to SWARM and Sven Gard methods.

Multiplex PCR was performed according to the protocol described by Norazah Ahmad et al. [11]. This assay provides a specific PCR product for the species Salmonella-429bp gene and the gene encoding the putative cation transporter in S. Paratyphi B for d-tartrate-positive strains (Java) -290bp. Our study gave the first molecular characterization of the Bulgarian S. Paratyphi B strains. After mPCR analysis, the results of capillary gel electrophoresis confirmed all tested strains as d-tartrate fermenting $(\mathrm{dT}+) \mathrm{S}$. Paratyphi B strains, also called Java (Figure 1). The application of genotypic methods to $108 \mathrm{~S}$. enterica serovar Paratyphi B strains isolated from diverse sources between 2002 and 2010 in Germany revealed that the serovar is polyphyletic. The three clonal complexes identified and characterized in this study were also recently detected within an international set of S. enterica serovar Paratyphi B strains based on MLST analysis [12]. 
We observed the following features during the clinical course of paratyphoid fever in our patients. In most children, watery diarrhea was the leading symptom, with constipation occurring only in $6.6 \%$ of the cases. The clinical characteristics are summarized in Table 1. Of all 33 cases of children with paratyphoid gastroenteritis, 30 had active diarrhea. There were only two children with constipation. Twenty-six patients had vomiting. They were all febrile. All children over the age of 5 had abdominal pain complaints. For the younger children, these subjective data are uncertain. Twenty-four of the children had a marked degree of dehydration, which required intravenous infusions as a symptomatic therapy. Eight children had pronounced anemic syndrome and 11 had leukopenia. One child had thrombocytopenia. In 30 patients, the C-reactive protein (CRP) was elevated. No child had rash. In patients from India hallmarks of paratyphoid fever include abdominal pain and high fever, with fever being the main presenting feature (as high as $75 \%$ of the cases) in the initial stages. Diarrhoea may be a presenting feature especially in immunocompromised cases and children. Classical clinical features of the disease were comparable among patients under and above 5 years of age but other manifestations, such as diarrhea, hepatomegaly, anemia and complications, are generally more frequent in children up to 5 years of age [13, 14]. In a French study from 1993 to 2015, 50 cases of paratyphoid fever among children and adolescents were identified. Only seven children $(14 \%)$ had no recent travel history. Illness was characterized by a history of fever combined with diarrhea in 48 patients $(96 \%)$. Constipation (6\%), rose spots $(1 \%)$, splenomegaly $(6 \%)$ and relative bradycardia (2\%) were not commonly reported [15].

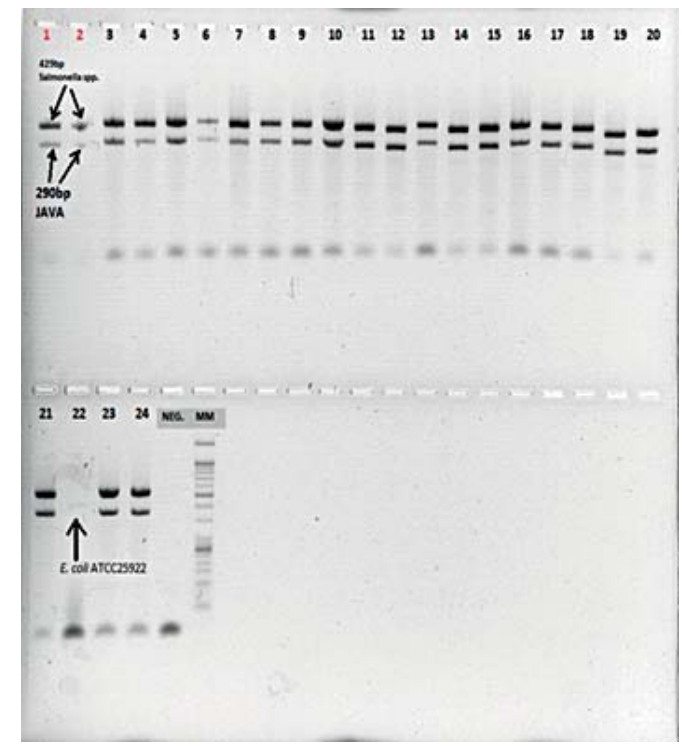

Fig. 1. Results of capillary gel electrophoresis. Line 22 negative control E. coli ATCC25922
Table 1. Clinical manifestations in 33 children

\begin{tabular}{|l|l|}
\hline Symptoms/Laboratory features & $\mathbf{N}(\%)$ of patients \\
\hline Fever & $33(100 \%)$ \\
\hline High grade fever $\left(>40^{\circ} \mathrm{C}\right)$ & $11(33.3 \%)$ \\
\hline Diarrhoea & $30(90 \%)$ \\
\hline Blood in stools & $21(70 \%)$ \\
\hline Constipation & $2(6.6 \%)$ \\
\hline Vomiting & $26(78.7 \%)$ \\
\hline Abdominal pain & $19(63.3 \%)$ \\
\hline Dehydration & $24(72.7 \%)$ \\
\hline Anaemia ${ }^{1}$ & $8(24.2 \%)$ \\
\hline Leucopenia $<4000 / \mathrm{mm}^{3}$ & $11(33.3 \%)$ \\
\hline Thrombocytopenia $<150.000 / \mathrm{mm}^{3}$ & $1(0.3 \%)$ \\
\hline CRP $>6$ mg/l & $30(90 \%)$ \\
\hline
\end{tabular}

${ }^{1}$ According to WHO definition of anaemia adjusted to age (http:// www.who.int/vmnis/indicators/haemoglobin pdf, accessed [17 July 2016]).

The treatment of the children from our study was symptomatic, with restoration of the loss from dehydration. When etiologically diagnosed, patients were treated with the antibiotics ceftriaxone or ciprofloxacin for a median duration of 7 days. In the French study cited a third-generation cephalosporin was administered as the first-line regimen to $47(94 \%)$ patients, of which $46(92 \%)$ received ceftriaxone for a median duration of 6 days at a median dose of $50 \mathrm{mg} / \mathrm{kg} / \mathrm{d}$. The second-line regimen included ciprofloxacin [15].

The European Centre for Disease Prevention and Control in last years reported increased resistance to some antibiotics like ciprofloxacin and nalidixic acid [16]. In our study the standard disk-based susceptibility testing method did not detect salmonella with reduced susceptibility to ciprofloxacin. Susceptibility to all antimicrobial agents used was detected in $30 / 33(90 \%)$ of the bacterial isolates. Three isolates were characterized by resistance to amikacin and gentamicin.

\section{CONCLUSION}

The annual reports of cases of paratyphoid fever among Bulgarian children indicate that a quick and accurate method for microbiological diagnosis of the causative agent is needed. Multiplex PCR allows for quick identification of Salmonella species and simultaneous discrimination of $\mathrm{S}$. enterica serotype Paratyphi B dT+ and dT- strains. According to the results obtained in our molecular study, we found that the most common cause of paratyphoid fever among children in Bulgaria is variant Java Salmonella Para- 
typhi B. Unlike the course in adults, the infection in children occurs mainly with diarrheal syndrome and severe dehydration.

The increase in antibiotic resistance of S. paratyphi isolates in travel-associated cases reflects the situation mainly in endemic countries but it is not a leading problem in Bulgaria.

Disclosure summary: The authors have nothing to disclose.

\section{Acknowledgments}

This work is funded by the National Science Fund Bulgaria contract DM 13/7 from 21 December, 2017.

\section{REFERENCES}

1. Joshi S, Wattal C, Sharma A, Prasad KJ. Mixed. Salmonella infection - a case report. Indian J Med Microbiol 2002; 20:113-114.

2. Cindy Shuan Ju Teh, Kek Heng Chu, Kwai Lin Thong. Paratyphoid Fever: Splicing the Global Analyses. Int. J. Med. Sci. 2014; 11(7): 732-741.

3. Britto C, Pollard AJ, Voysey M, Blohmke CJ. An appraisal of the clinical features of pediatric enteric fever: systematic review and meta-analysis of the age-stratified disease occurrence. Clin Infect Dis 2017; 64:1604-11.

4. Gilman RH, Terminel M, Levine MM, et al. Relative efficacy of blood, urine, rectal swabs, bone marrow and rose spot cultures for recovery of Salmonella typhi in typhoid fever. Lancet 1975; 1:1211-1213.

5. Kristensen, M, Kauffmann F. Bakteriologische und klinische Erfahrungen über Infektionen mit d-weinsäurevergärenden Paratyphus B-bacillen. Z. Hyg. Infektionskr. 1937,120:149-154.
6. Kauffmann, F. Zur Differentialdiagnose und Pathogenitat von Salmonella java und Salmonella paratyphi B. Zeitschrijit $f \sim r$ Hygiene 1966, 141, 546-550.

7. Le Minor, L., Vernon, M., Popoff, M. Proposition Pour Une Nomenclature Des Salmonella. Annales De Microbiologie 133b, 1982,4, 245-254.

8. Ezquerra E., Burnens A., Jones C., Stanley J. Genotypic typing and phylogenetic analysis of Salmonella pavatyphi B and S. java with IS200. Journal of General Microbiology, 1993. $139,2409-2414$.

9. Kyung Ho Han, Seon Young Choi, Je Hee Lee, et. al. Isolation of Salmonella enterica subspecies enterica serovar Paratyphi $\mathrm{B} \mathrm{dT}+$, or Salmonella Java, from Indonesia and alteration of the d-tartrate fermentation phenotype by disrupting the ORF STM 3356. Journal of Medical Microbiology 2006. 55, 1661-1665.

10. Clinical resistance and clinical breakpoints. Standard Operating Procedure. EUCAST definitions of clinical breakpoints and epidemiological cutoff values. 2018. http://www.eucast. org/fileadmin/src/media/PDFs/EUCAST_files/EUCAST_ SOPs/2018/EUCAST_SOP_3.2_Breakpoint_Review_and_ Revision_20180123.pdf.

11. Norazah Ahmad, Sh. Tang Gee Hoon, M. K. Abd Ghani, Koh Yin Tee. The discrimination of d-tartrate positive and d-tartrate negative $S$. enterica subsp. enterica serovar Paratyphi B isolated in Malaysia by phenotypic and genotypic methods. Malaysian J Pathol. 2012;34(1):35-39.

12. Achtman M, Wain J, Weill $F X$ et al. Multilocus sequence typing as a replacement for serotyping in Salmonella enterica. PLoS Pathog. 8:e1002776. doi:10.1371/journal.ppat.1002776.

13. Walia M, R Gaind, P Paul, et al. Age related clinical and microbiological characteristics of enteric fever in India. Trans $\mathrm{R}$ Soc Trop Med Hyg 2006;100: 942-948.

14. Sinha A, Sazawal S, Kumar R, et al. Typhoid and paratyphoid fever in children aged less than 5 years. Lancet, 1999;354:734-37.

15. Connor BA, Schwartz E. Typhoid and paratyphoid fever in travellers. Lancet Infect Dis 2005; 5:623-8.

16. Antimicrobial resistance in zoonotic bacteria still high in humans, animals and food, say ECDC and EFSA. 2018. https:// ecdc.europa.eu/sites/portal/files/documents/Press\%20release_ECDC\%20EFSA_AMR\%20zoonoses\%202016.pdf 